\section{Diagnostic Analysis of Silicon Strips Detector Readout in the ATLAS Semi-Conductor Tracker Module Production}

\author{
Alessandra Ciocio ${ }^{\mathrm{a}}$
}

\author{
representing the ATLAS SCT Collaboration
}

Formatted: Top: $1.25 "$ "Bottom:

$1.44^{\prime \prime}$

Deleted: Diagnostic Tools for

Deleted: during

${ }^{a}$ Lawrence Berkeley National Laboratory, 1 Cyclotron Road, Berkeley, California, 94720, USA

\title{
Abstract
}

The ATLAS Semi-Conductor Tracker (SCT) Collaboration is currently in the production phase of fabricating and testing silicon strips modules for the ATLAS detector at the Large Hadron Collider being built at the CERN laboratory in Geneva, Switzerland.

A small but relevant percentage of ICs developed a new set of defects after being mounted on hybrids that were not detected in the wafer screening. To minimize IC replacement and outright module failure, analysis methods ,were developed to study IC problems during the production of SCT modules.

These analyses included studying wafer and hybrid data correlations to finely tune the selion of to utilize the ability to adjust front-end parameters of the IC in order to reduce the rejentate of fabricated components.

This paper will discuss a few examples of the problems encountered during the production of SCT hybrids and

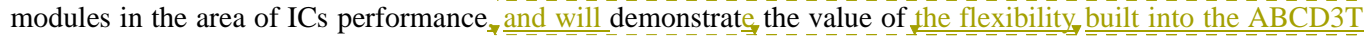
chip

(C) 2001 Elsevier Science. All rights reserved

Keywords: ATLAS SCT; silicon detector; IC; ABCD3T; 


\section{Introduction}

The ATLAS Semi-Conductor Tracker SCT [1] will consist of 4 cylindrical layers (barrel) in the central region of the Inner Detector [2], at 300, 373, 447, and $520 \mathrm{~mm}$ radii from the beam line, and 9 end-cap disks on each side of the barrel detector. The barrel and end-cap are populated with 4,088 silicon strips modules [3] [4] with a total area of silicon of $61 \mathrm{~m}^{2}$. The ATLAS SCT module is built with two pairs of identical, single-sided silicon micro-strip sensors (768 AC-coupled, p-strips on ntype silicon, $80 \mu \mathrm{m}$ strips pitch) glued back-to-back, with a 40 mrad stereo angle on a mechanical core (baseboard) made of thermal pyrolitic graphite (TPG) to transfer the heat generated by the readout electronics and sensors to the cooling pipe.

Each pair of strip sensors is wire-bonded to form double-long strips of $126 \mathrm{~mm}$ length. A hybrid flexible circuit made of copper/polyimide laminate is wrapped around the two sides of the sensors at the center of the module, bridged over the sensors.

The front-end readout of the SCT is handled by ASICs realized in the radiation hard DMILL technology as will be discussed in the next section. There are 12 ASICs on a module and they are glued and bonded on the hybrid, 6 on each side of the module. The full SCT requires $\sim 50,000$ ICs for a total of 6.2 million readout channels.

ASICs were tested on wafer at three institutes (CERN, Rutherford Appleton Lab and the Santa Cruz Institute for Particle Physics). During the wafer screening the ASICs were tested to verify their analogue front-end performance, such as gain and noise level, and their digital functions. They were graded according to electrical specifications and then picked (diced) to be used on modules.

The production of the barrel modules is carried out by clusters of institutes from Japan, Scandinavia, UK and the USA. The, end-cap. modules are produced in Australia, Germany, Netherlands, Spain, Switzerland and the UK. Barrel module production is almost completed and the end-cap is underway. All modules need to satisfy several mechanical and electrical specifications and are classified following a highly controlled quality assurance process to achieve the same quality of production across multiple assembly sites. Similarly to the wafer screening, the front-end readout electronic on hybrid is tested for the uniformity of the threshold, response in the gain, timing, noise levels and noise occupancy per channel. The testing method is presented in section 3 .

During the module production phase, a small but relevant percentage of the ASICs mounted on hybrids, -but graded-to-be- within-the "perfect chip" specifications at the wafer level, developed new set of defects. Some of these unexpected defects turned out to be due to a higher sensitivity for some of the ASICs to small differences in the operating conditions. As discussed in section 4, this led to the development of dedicated analyses which took advantage of the flexibility built into the ASIC to adjust its front-end parameters, and included the correlation studies of the module response with the results obtained during the wafer screening.

In section 5 we present the studies performed in the case of two defects observed in the ASICs after being mounted on hybrids, Solutions were found that allowed us to keep using those chips. During the module production it was important to minimize IC replacement and outright module failure. These studies we are also demonstrating the value of the flexibility built into the ABCD3T chip.

$\mathbf{x}$

\section{The ATLAS SCT Readout electronics}

The front-end readout of the SCT consists of ASICS realized in the radiation hard DMILL technology, named ABCD3T [5], featuring a 128channel analogue front-end consisting of amplifiers and comparators, and a digital readout circuit, and operating at the LHC bunch crossing frequency of $40 \mathrm{MHz}$. The ABCD3T chip utilizes a "binary" scheme where the signals from the silicon detector are amplified and then compared to a threshold. Only the result of this comparison, based on a hit or no-hit logic, enters the input register and the digital pipeline. The channel-to-channel matching, although acceptable for the non-irradiated chips, is expected to deteriorate significantly in the lifetime

\begin{tabular}{l} 
Formatted: Right: $-0.06 "$ \\
\hline Formatted: Font: $12 \mathrm{pt}$ \\
\hline Formatted: Font: $12 \mathrm{pt}$ \\
\hline Formatted: Font: $12 \mathrm{pt}$ \\
Formatted: Font: $12 \mathrm{pt}$ \\
Formatted: Font: $12 \mathrm{pt}$ \\
Formatted: Font: $12 \mathrm{pt}$ \\
\hline
\end{tabular}

\begin{tabular}{|l|}
\hline Deleted: B \\
\hline Deleted: E \\
\hline Deleted: C \\
\hline
\end{tabular}

\begin{tabular}{|c|}
\hline Formatted: Font: Times New Roman \\
\hline $\begin{array}{l}\text { Deleted: this article we describe the } \\
\text { ATLAS SCT module and Front-End } \\
\text { readout, the testing method, and } t\end{array}$ \\
\hline Deleted: he \\
\hline Deleted: diagnostic tools \\
\hline $\begin{array}{l}\text { Deleted: developed to study a new set } \\
\text { of }\end{array}$ \\
\hline Deleted: of \\
\hline Deleted: IC \\
\hline Deleted: ' \\
\hline Deleted: \\
\hline Deleted: during the hybrid/mod $\ldots$ [1] \\
\hline Deleted: The full SCT requires $\ldots$ [2] \\
\hline Formatted: Font: $12 \mathrm{pt}$ \\
\hline Formatted: Font: $12 \mathrm{pt}$ \\
\hline Deleted: . \\
\hline Formatted: Font: Times New Roman \\
\hline Formatted: Right: $-0.07 "$ \\
\hline Deleted: module and \\
\hline Deleted: The ATLAS SCT mod _.. [3] \\
\hline Deleted: F \\
\hline Deleted: E \\
\hline Deleted: is handled by \\
\hline Deleted: ABCD3T \\
\hline Deleted: \\
\hline Deleted: operates \\
\hline Formatted: Font: $12 \mathrm{pt}$ \\
\hline Deleted: ABCD3T \\
\hline Deleted: the \\
\hline Formatted: Font: $12 \mathrm{pt}$ \\
\hline Formatted: Font: $12 \mathrm{pt}$ \\
\hline Formatted: Font: $12 \mathrm{pt}$ \\
\hline
\end{tabular}


of the experiment. To ameliorate this effect, an internal 4-bit trim DAC controlling the comparator offset is used for each channel [6]. The DAC has 4 selectable ranges. Because the optimum values of the currents required by the front-end amplifier decrease with irradiation, two 5-bit DACs are also implemented in the chip to adjust the operating point of the front-end amplifier: one to set the current in the front-end transistor (Preamp current) and the other to set the bias of the second stage amplifier and comparator (Shaper current or Ish),

The ABCD3T chip also contains a dedicated internal charge injection circuit to calibrate the chip. A voltage step across the calibration capacitor produces a charge being injected into the front-end of the chip with variable amplitude controlled by an 8-bit DAC which could range from 0 to $16 \mathrm{fC}$.

The calibration pulses are issued with a delay (Strobe Delay) relative to the clock. It is necessary to adjust the Strobe Delay for each chip to account for variations in the propagation delay of the integrator-comparator.

Wafers of 256 IC's were fabricated by ATMEL (Nantes, France).

Beyond the wafer fabrication by the foundry, the collaboration took responsibility for the rest of the production process. The various tasks were split over many institutes in order to share the work among the available resources. The wafer testing was shared by three institutes (CERN, Rutherford Appleton Lab and the Santa Cruz Institute for Particle Physics). The wafer sawing and chip picking was performed by third party vendors.

The complexity of the ABCD3T chip demanded the design and implementation of a sophisticated tester [7] to verify the analogue front-end performance (gain and noise), the digital functions (control register, addressing, communication, pipeline, and output buffer), the power consumption, the linearity of the DACs, and the I/O Signals properties (timing, amplitudes, and duty cycles). The testing rate was 5 hours/wafer.

ASICs were graded into the following main classifications: a) Perfect (meets all specifications) b) 1 bad-channel (IC meets all specifications except for 1 channel) c) 2 bad-channel (IC meets all specifications except for 2 channels) d) Digitally perfect (passes all digital tests except perhaps full ${ }^{3}$ pipeline and has more than 2 bad channels) e) Bad (all else).

Tests of 1-bad-channel ICs in modules including irradiations have not shown any extra reliability problems beyond the loss of 1 channel.

\section{SCT Hybrid Testing: method and typical results}

An extensive suite of both hardware and software [8] has been developed to facilitate hybrid and module testing. The readout system is based on custom designed VME boards including low voltage and high voltage boards [9] [10] developed specifically for SCT production testing.

Two automated series of tests have been designed to simplify the testing procedure: a) characterization sequence aimed to perform a full characterization of a hybrid or a module for both digital and analogue performance and b) confirmation sequence with a reduced set of tests to ensure the basic digital and analogue functionality of the chips. The performance of the analogue part of the chip for each channel, such as gain, offset, noise, is tested by using the internal calibration circuit of the ABCD3T chip.

The basic method for the characterization of the analog circuitry of the ABCD3T chip consists of an efficiency scan for different discriminator threshold values with a fixed calibration charge. A typical "S-curve" from this measurement is shown in fig. 1 , fitted to a complementary error function. The 50\% point (vt50) indicates the threshold value, which corresponds to the injected charge after amplification, and the width characterizes the output_noise.
Deleted: ...FE ........The two parameters....are... called ... (Ish) ... [4]

Deleted: M...t...r .. [5]

Deleted: ABCD3T...performance ...of the analogue part by issuing calibration pulses with variable amplitude

Deleted: L...V...H...V

Formatted: Font: Times

Deleted: and ... delay

Formatted

Deleted: the ...C...S...e ...;...the C...S... is ...ABCD3T ... [10]

Deleted: There are 12 ASIC's on module and they are glued and bonded on the hybrid, 6 on each side of the module. 9 $<\#>$ The Wafer production testing and IC grading $\uparrow$

Deleted: the ...W...W [11]

Deleted: ABCD3T...,...

Deleted: ABCD3T...\& ...P [13]

Deleted: $-\ldots-\ldots$ P [14] 
4

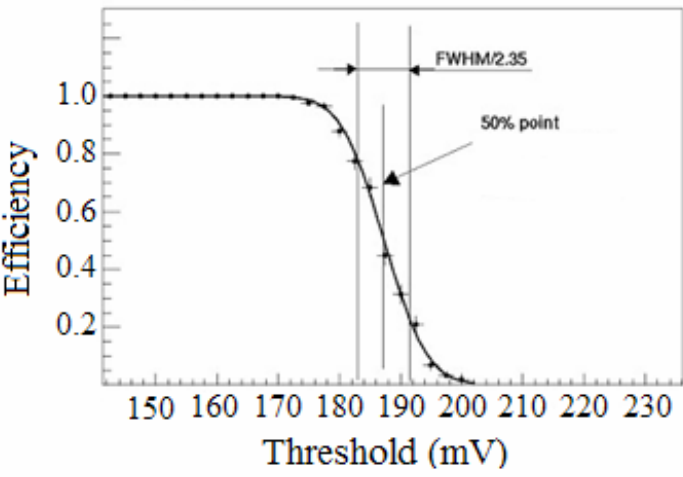

Fig.1. Example of a threshold scan for a singe channel with fixed injected charge. The midpoint of the transition gives the value of the threshold equal to the injected pulse after amplification. The width of the transition measures the electronic noise. The histogram is fitted to a complementary error function.

To measure the gain and offset, we perform a scan at three different calibration input charges (from $1.5 \mathrm{fC}$ to $2.5 \mathrm{fC}$ ) in the confirmation sequence and ten charge values (from $0.5 \mathrm{fC}$ to $8 \mathrm{fC}$ ) in the characterization sequence. The set of vt50 points versus calibration charge amplitude is the response curve of each channel. The gain is determined by the slope of a polynomial fit to the response curve, the offset is the intercept at zero charge injection and the input noise is determined by dividing the output noise by the gain. A typical set of plots that are obtained with a full characterization is shown for the 768 channels of the top side of a module (6 chips, 128 channels/chip) in fig. 2. Prior to the efficiency scan the optimal delay (Strobe Delay) of the input charge with respect to the clock for every chip must be determined. This is obtained with a Strobe Delay scan performed as the very first of the analogue test sequence to determine the correct Strobe Delay setting which is used during the analogue tests. A $4 \mathrm{fC}$ signal at $2 \mathrm{fC}$ threshold is injected (using the internal calibration circuit) as the Strobe Delay is varied. A fit is made to both the rising edge and the falling edge of the signal to find the points at which the $50 \%$ efficiency is achieved.

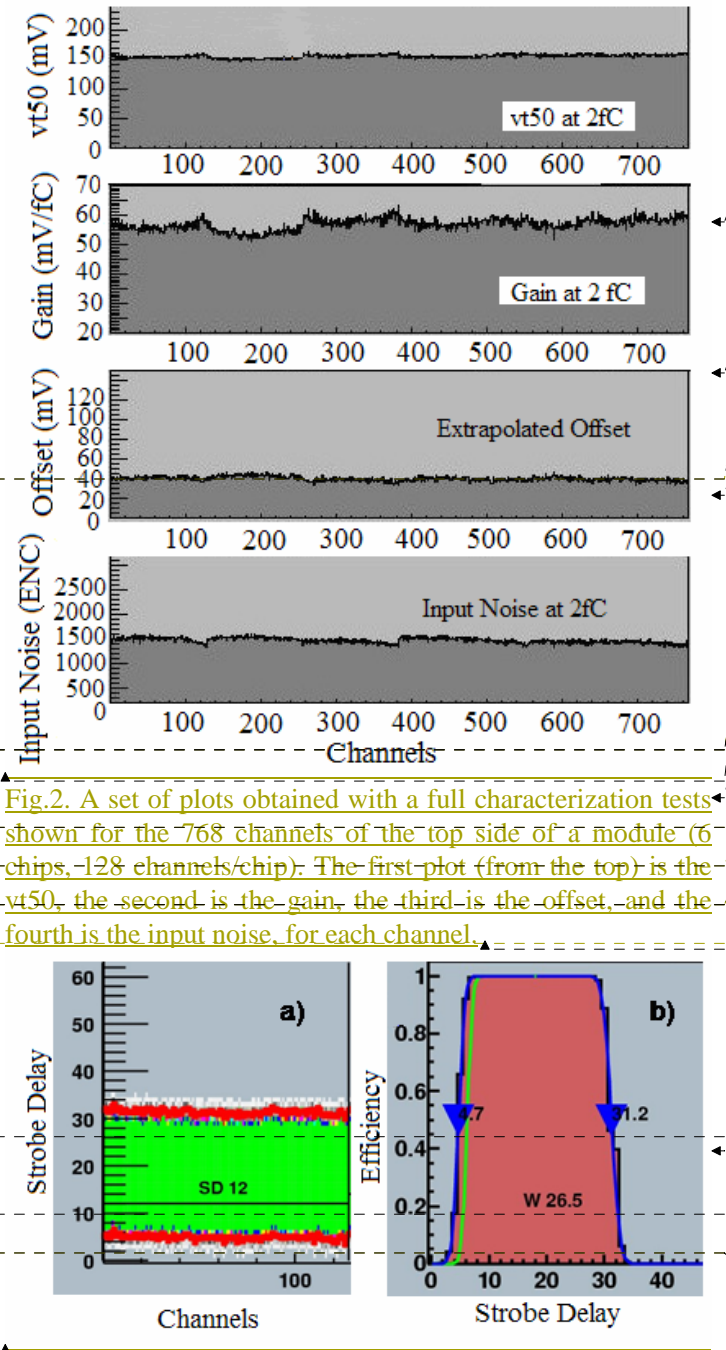

Fig.3. a) Strobe Delay at the $25 \%$ of the distance between minimum and maximum delay (horizontal line); b) the fit to both the rising edge and the falling edge of the signal to find the points at which the $50 \%$ efficiency is achieved as the Strobe Delay varies.

The Strobe Delay is then taken at the $25 \%$ of the distance between those two points with the reference to the rising edge of the Strobe Delay peak which correspond to the falling edge of the signal. Fig. 3a shows an example of a Strobe Delay plot and fig. 3b shows the signal efficiency fit for

\begin{tabular}{|c|}
\hline Deleted: ๆ \\
\hline Formatted: Indent: First line: 0" \\
\hline Formatted: Font: $12 \mathrm{pt}$ \\
\hline Formatted: Font: $12 \mathrm{pt}$ \\
\hline Formatted: Indent: First line: 0" \\
\hline $\begin{array}{l}\text { Formatted: Text, Right: 0", Tabs: } \\
\text { Not at 3" }\end{array}$ \\
\hline Deleted: do the \\
\hline Formatted: Font: $12 \mathrm{pt}$ \\
\hline Formatted: Font: $12 \mathrm{pt}$ \\
\hline $\begin{array}{l}\text { Formatted: Els-body-text, Left, } \\
\text { Indent: First line: 0", Right: 0", } \\
\text { Adjust space between Latin and Asian } \\
\text { text, Adjust space between Asian text } \\
\text { and numbers, Tabs: } 3 \text { ", Left }\end{array}$ \\
\hline Deleted: C \\
\hline Formatted: Font: $12 \mathrm{pt}$ \\
\hline Deleted: points \\
\hline Formatted: Font: $12 \mathrm{pt}$ \\
\hline Deleted: C \\
\hline Formatted: Font: $12 \mathrm{pt}$ \\
\hline $\begin{array}{l}\text { Formatted: Font: Times New } \\
\text { Roman, } 12 \text { pt }\end{array}$ \\
\hline Deleted: $50 \%$ \\
\hline $\begin{array}{l}\text { Formatted: Font: Times New } \\
\text { Roman, } 12 \mathrm{pt}\end{array}$ \\
\hline Deleted: (vt50) \\
\hline Formatted: Font: Times, $10 \mathrm{pt}$ \\
\hline Formatted: Font: $12 \mathrm{pt}$ \\
\hline $\begin{array}{l}\text { Formatted: Indent: First line: } 0 " \text {, } \\
\text { Tabs: }-0.38 ", \text { Left }\end{array}$ \\
\hline Formatted: Font: $12 \mathrm{pt}$ \\
\hline Formatted: Font: $12 \mathrm{pt}$ \\
\hline Deleted: figure \\
\hline Formatted: Font: 12 pt \\
\hline $\begin{array}{l}\text { Deleted: A "Strobe Delay Scan" is } \\
\text { performed as the very first of the } \\
\text { analogue test sequence to determ ... [15] }\end{array}$ \\
\hline Formatted: Font: $12 \mathrm{pt}$ \\
\hline Formatted: Indent: First line: 0" \\
\hline Formatted: Font: $12 \mathrm{pt}$ \\
\hline Formatted: Font: $12 \mathrm{pt}$ \\
\hline Formatted: Font: $12 \mathrm{pt}$ \\
\hline Formatted: Font: $12 \mathrm{pt}$ \\
\hline Formatted: Font: $12 \mathrm{pt}$ \\
\hline Formatted: Font: 12 pt \\
\hline Formatted: Font: $12 \mathrm{pt}$ \\
\hline
\end{tabular}


one chip. The horizontal line in fig. 3a indicates the $25 \%$ of the distance from the minimum delay.

\section{Diagnostic Analysis Methods}

A small but relevant percentage $(<5 \%)$ of IC's that met all the specifications at the level of wafer testing, developed a new set of defects after being mounted on production hybrids. While defects in the digital circuitry were considered as fatal and the chips were replaced, some of the defects in the analogue circuitry were instead studied to see if the performance of the ASIC could be improved by adjusting its front-end parameters. Small differences in the operating conditions of the two testing phases (wafer screening and hybrid tests), turned out to be the primary reason for the appearance of some of the new defects. This fact helped in identifying the area of sensitivity of the defective chips that needed to be studied. As described in Section 2, because of degradation due to irradiation, the ABCD3T chip provides the feature of adjusting the operating point of the frontend parameters such as the preamplifier and the shaper current (Ish). For the same reason, the analogue and digital voltage can also be adjusted. The capability of adjusting the Strobe Delay was also used in the analysis of defective chips. By measuring the response versus the Strobe Delay we were able to determine the transit time and the rise time of each channel. This flexibility of the chip, turned out to be very important_during production testing and was used as a tool for the diagnostic analysis. Software was also developed to study the data from the wafer screening. Useful information for the studies was: various settings parameters: gain and noise response of the chips, analogue current drawn individually by every chip (Icc) when the analogue supply voltage is applied; and the front-end DACs values. Correlation studies between wafer screening quantities and the results obtained from the same chips mounted on hybrids were performed.

\section{Study of Defective ASIC}

Amongst those defects that were considered for study we had: a) a very large spread of gain in all channels, a defect classified as "Large Gain Spread" (LGS) (fig. 4) and b) a block of contiguous channels for which the measured gain is $10 \%$ to $20 \%$ lower than their neighbours and the measured input noise is high (fig. 5).

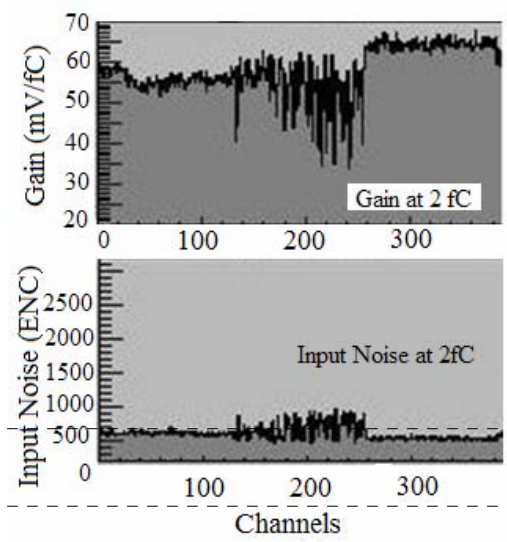

Fig.4. Example of LGS defect in the Gain (top) and the Noise (bottom). The second chip of the three chips shown here has the LGS defect.

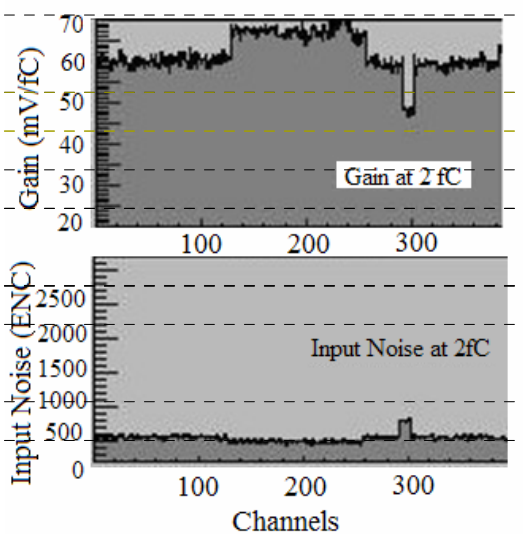

Fig.5. Exampte- of BEG-defeet in the Gain (top) and the-Noise (bottom). The third chip of the three chips shown here has the BLG defect.

This defect was called Block Low Gain (BLG). In the next two sections of this paper we will
Formatted: Bullets and Numbering

Deleted: The operating point for the Strobe Delay is chosen as the $25 \%$ of the distance between the $50 \%$ efficiency of

the rising edge and the falling edge of the $4 \mathrm{fC}$ signal at $2 \mathrm{fC}$ threshold whe ... [16]

Formatted: Bullets and Numbering

Deleted: A small but relevant ... [17]

Deleted: “

Deleted: "

Deleted: 3

Deleted: ;

Formatted: Indent: First line: $0.19 "$

Deleted: (BLG)

Deleted: 4

Formatted: Font: $12 \mathrm{pt}$

Formatted

Formatted: Font: $12 \mathrm{pt}$

Deleted: १

Formatted

Deleted: to

Deleted: F

Formatted

Deleted: E

Formatted

Deleted:

Formatted

Formatted

Formatted: Font: $12 \mathrm{pt}$

Deleted: , and the Strobe delay

Deleted: an

Deleted: tool

Formatted: Font: $12 \mathrm{pt}$

Formatted: Font: Times New Roman

Formatted: Font: Times New Roman

Formatted: Font: Times New Roman

Formatted: Font: $12 \mathrm{pt}$

Formatted: Font: $12 \mathrm{pt}$

Formatted: Font: $12 \mathrm{pt}$

Formatted: Font: $12 \mathrm{pt}$

Formatted: Font: $12 \mathrm{pt}$

Deleted: . I

Formatted

Formatted

Formatted: Font: $10 \mathrm{pt}$ 
discuss the above effects. Solutions were found that saved a large fraction of the chips which otherwise would have been replaced.

\subsection{Large Gain Spread (LGS)}

Fig. 4 shows an example of a chip with the Large Gain Spread (LGS) defect. We used the data from the wafer screening to perform a comparison between the gain response of the twelve chips on the hybrid and the same chips when tested on the wafer. The result is shown in fig. $6_{x \rightarrow}$

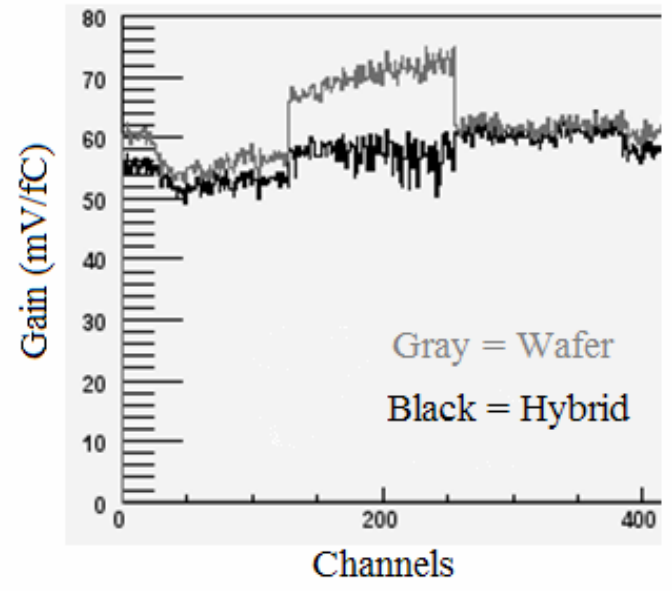

Fig. $\overline{6}$ Wafer gain data (gray) compared to hybrid gain data (black) for 3 chips. The second chip has LGS.

The large difference in the gain of the defective chip between the wafer and hybrid test suggests sensitivity to the small differences in the operating conditions of the two tests. Fig 7 shows the gain vs Ish for four chips which show LGS and four normal chips. Clearly the LGS chips are much more sensitive to changes in Ish. Fig. 8 shows the gain vs Vcc (the analogue supply voltage) for a LGS chip (chip 9 in the figure) for three values of Ish and for a normal chip (chip 0). The slope for the LGS chip is significantly greater at the nominal value of Ish $(30 \mu \mathrm{A})$ than at a lower value of Ish, Fig. 9 shows the rms of the gain in LGS chips and normal chips vs Ish. Lowering Ish to $20 \mu \mathrm{A}$ brought most of the LGS chips into a satisfactory operating region.

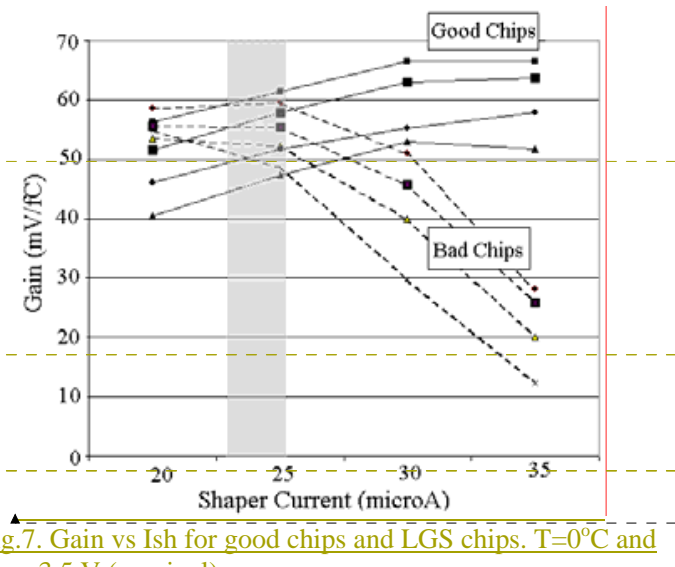

Fig.7. Gain vs Ish for

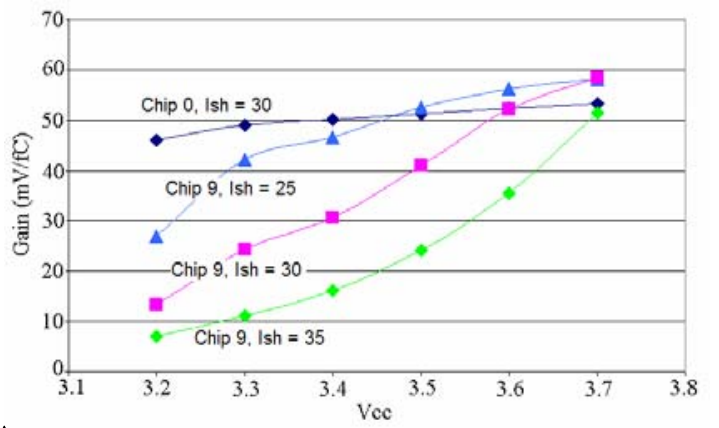

Fig.8. Gain vs VCC of a LGS chip (Chip 9) at different Ish settings compared to a good chip (Chip 0) at nominal Ish setting.

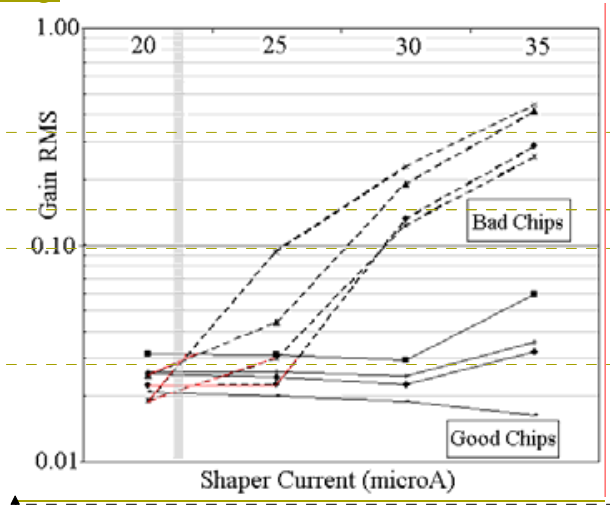

Fig.9. Gain RMS-vs Ish for gøod-chips and $\mathrm{E} G \mathrm{G}$ ehips. $\mathrm{T}=0^{\circ} \mathrm{C}$ and $V c c=3.5 \mathrm{~V}$ (nominal). The "gray" band indicates thee range of Ish at which the bad chips behave like good chips.
Formatted: Font: Times, $12 \mathrm{pt}$

Formatted: Indent: First line: $0.19^{\prime \prime}$

Deleted: as seen in hybrids and the diagnostic tools used to analyze the defective chips. The solutions saved a large fraction of them which otherwise would have been replaced. $\uparrow$

Formatted: Indent: Left: $0.25 "$

Formatted: Bullets and Numbering

Deleted: The

Deleted: first diagnostic tool that was used was the

Deleted: a

Deleted: 5

Deleted:

Formatted: Font: Times, $12 \mathrm{pt}$

Formatted: Font: 12 pt

Formatted: Font: $12 \mathrm{pt}$

Deleted: troubled

Deleted: sensitivity

Deleted: changes

Deleted: 6

Deleted: 7

\begin{tabular}{l} 
Deleted: nominal parameters \\
Deleted: ( \\
\hline Deleted: $=$ \\
\hline Deleted: a \\
Deleted: \\
\hline Deleted: $=25 \mu \mathrm{A}$ \\
\hline Deleted: 8
\end{tabular}


We also looked at the values of Icc of individual chips as measured on the wafer (on the hybrid we measure the overall Icc for all twelve chips) and we found a strong correlation between high Icc and chips with LGS. At the nominal value of the shaper current $(30 \mu \mathrm{A})$, Icc for chips with the LGS defect is typically $20 \%$ higher than average, but that was not considered enough of a deviation for rejecting the chips during the wafer screening. Fig. 10 is a distribution of Icc for all chips from one particular wafer. The two chips with higher Icc are the ones that later on showed the LGS effect on the hybrid.

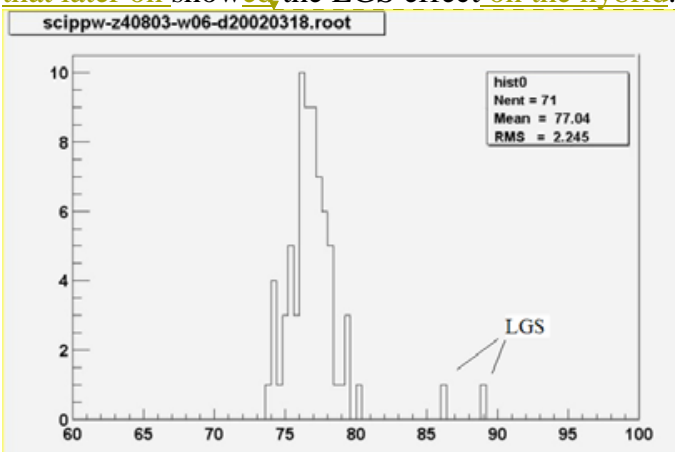

Fig.10. Distribution of Icc for chips coming from the same wafer.

We also analyzed the first 100 production hybrids and by looking at the deviation of Icc from the mean value of Icc per hybrid (fig. 11) we confirmed the strong correlation between higher Icc on wafers and LGS defects on hybrids.

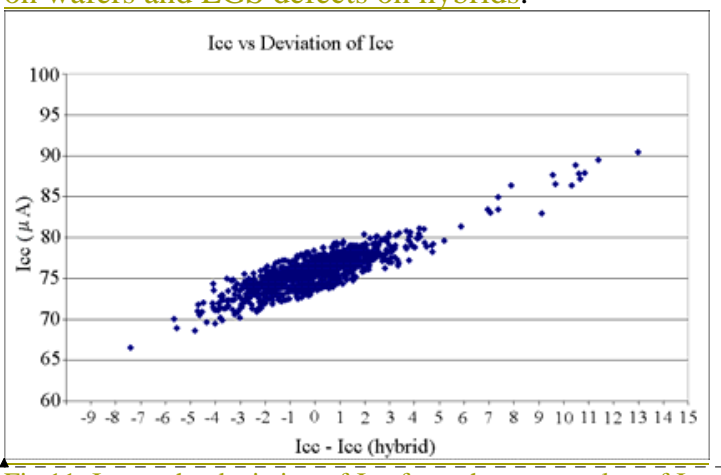

Fig.11. Icc vs the deviation of Icc from the mean value of Icc per hybrid for 100 production hybrids. All the chips known having the LGS defect correspond to the points above a deviation of $5 \mu \mathrm{A}$ and higher Icc.
As it could be seen in the same figure, there are 17 points with higher Icc and larger deviation from the mean Icc. Those points correspond to the chips with LGS defect.

A cut on Icc in the wafer data was then adopted to pre-select chips and to keep aside suspected chips with the LGS defect in the construction of subsequent hybrids. For the chips already used and that showed LGS we used a lower Ish setting, as demonstrated being a cure for this defect.

\subsection{Block Low Gain (BLG)}

The "Block Low Gain” (BLG) defect is seen as a block of continuous channels showing low gain and correspondingly higher noise (fig. 5). The noise was a critical issue and the reason for which this, defect needed to be addressed since these channels failed the nominal noise cut. The wafer/hybrid comparison for the same chip (in fig. 12 it is the ninth chip) shows on the contrary that this chip has a block of high gain channels.

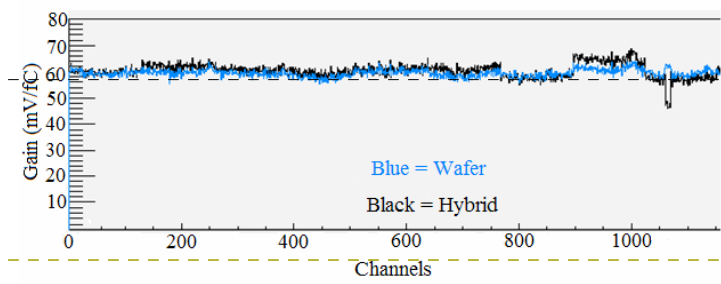

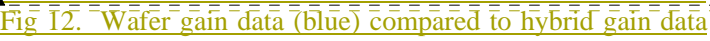
(black) for 9 chips. The ninth chip has BLG. This is the same as the third chip on fig5.

This finding suggests that this defect as seen on the hybrid might be explained with a timing problem. For the channels that have indeed a higher gain the pulse is likely to start in the preceding time bucket. Fig. 13 , shows the Strobe Delay plot for the three of the same group of chips of one side of the module (bottom side). The 3rd chip is the one with, BLG and the affected channels have a longer Strobe Delay indicating that they have a shorter propagation delay In the same figure the optimal Strobe-Delay is shown at the $40 \%$ of the plateau. Studies of the Strobe Delay were conducted on hybrids with chips showing the BLG effect.

\begin{tabular}{|c|}
\hline Deleted: $(30 \mu \mathrm{A})$ \\
\hline Deleted: ๆ \\
\hline Deleted: can \\
\hline Deleted: be used \\
\hline Deleted: 9 \\
\hline Formatted: Indent: Left: $0.25 "$ \\
\hline Formatted: Bullets and Numbering \\
\hline Deleted: ing \\
\hline Formatted: Font: $12 \mathrm{pt}$ \\
\hline Deleted: the \\
\hline Deleted: bothersome \\
\hline Deleted: that \\
\hline Deleted: e \\
\hline Deleted: s \\
\hline Deleted: our \\
\hline Formatted: Font: $12 \mathrm{pt}$ \\
\hline $\begin{array}{l}\text { Deleted: We found a strong correlation } \\
\text { between high Icc and chips with LGS }\end{array}$ \\
\hline Deleted: in 100 hybrids \\
\hline Deleted: 0 \\
\hline Formatted: Font: $12 \mathrm{pt}$ \\
\hline Formatted: Indent: Hanging: $0.13 "$ \\
\hline Deleted: This defect turned out to b \\
\hline Deleted: e \\
\hline Deleted: because \\
\hline $\begin{array}{l}\text { Deleted: and, with the standard strobe } \\
\text { delay, }\end{array}$ \\
\hline Deleted: s \\
\hline Deleted: 1 \\
\hline Deleted: is \\
\hline Deleted: s \\
\hline Deleted: d \\
\hline Deleted: 6 \\
\hline Deleted: chips \\
\hline Deleted: has \\
\hline Deleted: s \\
\hline Deleted: $\mathrm{d}$ \\
\hline Deleted: . \\
\hline Formatted: Font: Symbol \\
\hline Formatted: Indent: First line: $0.13 "$ \\
\hline
\end{tabular}


8

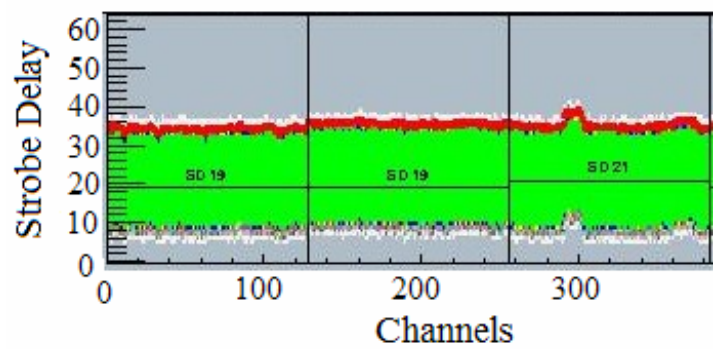

Fig.13. Strobe Délay plot for 3 chips. The horizontal line (per each chip) indicates the $40 \%$ from the rising edge of the Strobe Delay peak which correspond to the falling edge of the signal.

Fig. 14 shows (a) the gain and (b) the input noise as a function of the Strobe Delay averaged over 10 affected channels and 10 normal channels. The channels with the BLG defect reach a normal condition at a longer Strobe Delay and a significantly higher gain. The excess noise at the nominal Strobe Delay is apparently an artefact of using a too short Strobe Delay and jit disappears as the Strobe Delay is increased.
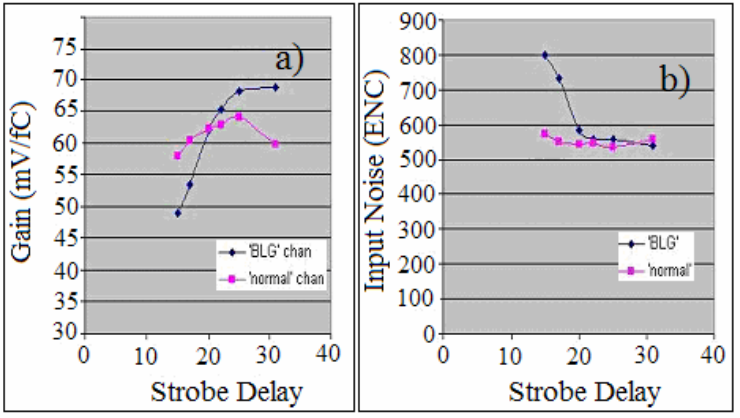

Fig.14. Gain and Input Noise vs Strobe Delay.

A hybrid built at UCSC with 2 chips showing this problem was irradiated with $2 \times 10^{14} \mathrm{n} / \mathrm{cm}^{2}$ (1 $\mathrm{MeV}$ NIEL equivalent in $\mathrm{Si}$ ) in the reactor in | Ljubljana [11]. By comparing the Strobe Delay plots before and after irradiation we concluded that the radiation slows down the chip causing the Strobe Delay to decrease uniformly. The 'BLG' channels then become normal, with the standard Strobe Delay after irradiation. The symptoms improve when Ish is lowered for the same reason.
About $2 \%$ of the chips used show the BLG defect and they can be used with a longer Strobe Delay.

\section{Conclusions}

During the SCT module production we were able to reduce the rate of ASIC replacements on hybrids with defective ASICs and increase the number- of - working modules. - - The- diagnostic analysis developed to study defective chips made use primarily of the flexibility built into the chip to adjust jits front-end parameters, demonstrating the value to have such capability It was very useful to also have, access to the detailed database containing the results from the wafer screening.

\section{Acknowledgments}

The ATLAS SCT is an effort made possible by a very large community from many institutions in Japan, UK, Scandinavia, USA, and Spain. I would like to acknowledge Y. Unno from KEK, P.Phillips from RAL, Alex Grillo from Santa Cruz and W. Dabrowski from FPNT UMM, Cracow, Poland for invaluable discussion and suggestions.

In particular I would like to thank Robert Ely from UC Berkeley and LBNL. The development of the analysis methods presented in this paper would not have been possible without him.

Furthermore, I acknowledge the support of the United States Department of Energy, and the United States National Science Foundation, and all the other funding authorities of the collaborating institutes.

\section{References}

[1] The ATLAS collaboration. Technical proposal for a general-purpose pp experiment at the LHC at CERN. Technical report, CERN, LHCC 94-43.

[2] The ATLAS Collaboration. ATLAS Inner detector technical design report.

Technical report, CERN, LHCC 99-14/15, 1999.

[3] T. Kondo et al., Construction and performance of the ATLAS silicon micro-strip barrel modules. Nucl. Instrum. Meth. A485 (2002) 27-42.
Formatted: Font: $12 \mathrm{pt}$

Formatted: Bullets and Numbering
Deleted: By developing diagnostic tools ...d...ies...have demonstrated the importance of the capability ...the ...FE and...the...importance of

having...the...from...tests ... [27]

Deleted: $2 \ldots . .$. s...d...The plateau in gain is not as flat as one might like in both case and

t...'...'...plateau...s...d...s...d...a s...d...becomes the same for all channels s...d ... [28]

Deleted: tools

Deleted: s...d...and ...es...s...d...and that moved... t...enough... so that they are happy ...s...d...Ish ... [29] 
[4] G. F. Moorhead et al., Detector modules for the endcaps of the ATLAS semiconductor tracker,

Nucl. Instrum. Meth. A485 (2002) 43-53.

[5] W. Dabrowski et al., Design and performance of the ABCD chip for the binary readout of silicon strip detectors in the ATLAS semiconductor tracker,

IEEE Trans. Nucl. Sci. 47 (2000) 1843-1850.

[6] W. Dabrowski, F. Anghinolfi, P. Jarron, J. Kaplon, S. Roe, P. Weilhammer, et al, "Radiation Hardness of the ABCD Chip for the Binary Readout of Silicon Strip Detectors in the ATLAS Semiconductor Tracker", in Proc. of the Fifth Workshop on Electronics for LHC Experiments, Snowmass, Colorado, USA, 1999, pp. 113-117.

[7] F. Anghinolfi et al., ASIC Wafer Test System for the ATLAS Semiconductor Tracker Front-End Chip,

IEEE Trans.Nucl.Sci.49:1080-1085, 2002.

[8] P.W. Phillips, G.F. Moorhead, et al.,

http://scttestdaq.home.cern.ch/sct-testdaq/sctdaq/sctdaq.html

[9] J. Bohm et al., SCT LV3

Institute of Physics, Academy of Science, The Czech Republic,

http://www-hep2.fzu.cz/Atlas/WorkingGroups/Projects/MSGC.html

[10] E. Gornicki et al., SCT HV Model HV203 User Manual, Institute of Nuclear Physics (IFJ), Cracow, Poland, http://chall.ifj.edu.pl/atlas/SCTHV/SCTHV PAJ.html

[11] http://www-f9.ijs.si/ mandic/ChipIrrad/spo233/spo233.html 
Deleted: Figure Captions

Fig.1. Example of a threshold scan for a singe channel with fixed injected charge.

This is a basic method to study the performance of a binary chip. The

midpoint of the transition gives the value of the threshold equal to the injected pulse after amplification. The width of the transition measures the electronic noise. The histogram is fitted to a complementary error function. $₫$

Fig.2. A set of plots obtained with a full characterization of a module. The first plot (from the top) is the vt50, the second is the gain, the third is the offset, and the fourth is the input noise, for each channel (768 channels in these plots). 1

I

Fig.3. Example of LGS defect in the Gain (top) and the Noise (bottom). 9

I

Fig.4. Example of BLG defect in the Gain (top) and the Noise (bottom).

वा

Fig.5. Wafer gain data (grey) compared to Hybrid gain data (black) for 12 chips.

Fig.6. Gain vs Ish for good chips and LGS chips. $\mathrm{T}=0^{\circ} \mathrm{C}$ and $\mathrm{Vcc}=3.5 \mathrm{~V}$ (nominal). 9

Fig.7. Gain vs Vcc of a LGS chip (Chip 9) at different Ish settings compared to a good chip (Chip 0) at nominal Ish setting.

Fig.8. Gain RMS vs Ish for good chips and LGS chips. $\mathrm{T}=0^{\circ} \mathrm{C}$ and $\mathrm{Vcc}=3.5 \mathrm{~V}$ (nominal).ๆ

Fig.9. Distribution of Icc for chips coming from the same wafer. 9

Fig.10. Icc vs the deviation of Icc from the mean value of Icc per hybrid. All the chips known having the LGS defect can be identified in the points above a deviation of 5.9

I

Fig.11. Strobe delay plot for 6 chips. 9

Fig.12. Gain and Input Noise vs Strobe Delay. 
Page 2: [1] Deleted

A. Ciocio

10/ 28/ 2004 4:36:00 PM

during the hybrid/module production phase

Page 2: [2] Deleted

A. Ciocio

10/ 28/ 2004 10:54:00 AM

The full SCT requires $~ 50,000$ ICs for a total of 6.2 million readout channels.

Page 2: [3] Deleted

A. Ciocio

10/ 28/ 2004 10:52:00 AM

The ATLAS SCT module is built with 2 pairs of identical, single-sided silicon microstrips sensors (768 AC-coupled, p-strips on n-type silicon, $80 \mu \mathrm{m}$ strips pitch) glued back-to-back, with a 40 mrad stereo angle on a mechanical core (baseboard) made of thermal pyrolitic graphite (TPG) to transfer the heat generated by the readout electronics and sensors to the cooling pipe.

Each pair of strips sensors is wire bonded to form double-long strips of $126 \mathrm{~mm}$ length. A hybrid flexible circuit made of copper/polyimide laminate is wrapped around the two sides of the sensors at the center of the module, bridged over the sensors.

\begin{tabular}{lll}
\hline Page 3: [4] Deleted & A. Ciocio & $10 / 27 / 2004$ 10:44:00 PM \\
\hline $\begin{array}{l}\text { Page 3: [4] Deleted } \\
\text { FE }\end{array}$ & A. Ciocio & $10 / 27 / 2004$ 10:50:00 PM \\
\hline $\begin{array}{l}\text { Page 3: [4] Deleted } \\
\text { Page 3: [4] Deleted } \\
\text { The tWo parameters }\end{array}$ & A. Ciocio & $10 / 27 / 2004$ 10:53:00 PM \\
\hline $\begin{array}{l}\text { Page 3: [4] Deleted } \\
\text { are }\end{array}$ & A. Ciocio & $10 / 27 / 2004$ 10:53:00 PM \\
\hline $\begin{array}{l}\text { Page 3: [4] Deleted } \\
\text { Called }\end{array}$ & A. Ciocio & $9 / 23 / 2004$ 10:23:00 PM \\
\hline $\begin{array}{l}\text { Page 3: [4] Deleted } \\
\text { (Ish) }\end{array}$ & A. Ciocio & $9 / 23 / 2004$ 10:24:00 PM \\
\hline $\begin{array}{l}\text { Page 3: [5] Deleted } \\
\text { Pas }\end{array}$ & A. Ciocio & $9 / 23 / 2004$ 10:25:00 PM \\
\hline
\end{tabular}

M

Page 3: [5] Deleted

A. Ciocio

10/ 28/ 2004 4:52:00 PM

t 


\begin{tabular}{|c|c|c|}
\hline $\begin{array}{l}\text { Page 3: [6] Deleted } \\
\text { ABCD3T }\end{array}$ & A. Ciocio & $10 / 7 / 2004$ 10:48:00 AM \\
\hline $\begin{array}{l}\text { Page 3: [6] Deleted } \\
\text { performance }\end{array}$ & A. Ciocio & 9/23/ 2004 10:20:00 PM \\
\hline $\begin{array}{l}\text { Page 3: [6] Deleted } \\
\text { of the analogue p }\end{array}$ & $\begin{array}{l}\text { A. Ciocio } \\
\text { ation pul }\end{array}$ & $\begin{array}{l}\text { 9/ 23/ } 2004 \text { 10:20:00 PM } \\
\text { amplitude }\end{array}$ \\
\hline $\begin{array}{l}\text { Page 3: [7] Deleted } \\
\quad \text { L }\end{array}$ & A. Ciocio & $10 / 27 / 2004$ 11:12:00 PM \\
\hline $\begin{array}{l}\text { Page 3: [7] Deleted } \\
\text { V }\end{array}$ & A. Ciocio & 10/ 27/ 2004 11:12:00 PM \\
\hline $\begin{array}{l}\text { Page 3: [7] Deleted } \\
\text { H }\end{array}$ & A. Ciocio & $10 / 27 / 2004$ 11:12:00 PM \\
\hline $\begin{array}{l}\text { Page 3: [7] Deleted } \\
\text { V }\end{array}$ & A. Ciocio & 10/27/2004 11:12:00 PM \\
\hline $\begin{array}{l}\text { Page 3: [8] Deleted } \\
\text { and }\end{array}$ & A. Ciocio & 10/ 4/ 2004 10:38:00 PM \\
\hline $\begin{array}{l}\text { Page 3: [8] Deleted } \\
\text { delay }\end{array}$ & A. Ciocio & 10/ 5/ 2004 1:43:00 PM \\
\hline $\begin{array}{l}\text { Page 3: [9] Formatted } \\
\text { Font color: Auto }\end{array}$ & A. Ciocio & 10/5/2004 1:40:00 PM \\
\hline $\begin{array}{l}\text { Page 3: [9] Formatted } \\
\text { Font color: Auto }\end{array}$ & A. Ciocio & 10/ 5/ 2004 1:40:00 PM \\
\hline $\begin{array}{l}\text { Page 3: [9] Formatted } \\
\text { Font: Times }\end{array}$ & A. Ciocio & 10/ 5/ 2004 1:40:00 PM \\
\hline $\begin{array}{l}\text { Page 3: [10] Deleted } \\
\text { the }\end{array}$ & A. Ciocio & $10 / 27 / 2004$ 11:13:00 PM \\
\hline $\begin{array}{l}\text { Page 3: [10] Deleted } \\
\quad \text { C }\end{array}$ & A. Ciocio & $10 / 27 / 2004$ 11:13:00 PM \\
\hline $\begin{array}{l}\text { Page 3: [10] Deleted } \\
\quad \text { S }\end{array}$ & A. Ciocio & $10 / 27 / 2004$ 11:13:00 PM \\
\hline $\begin{array}{l}\text { Page 3: [10] Deleted } \\
\text { e }\end{array}$ & A. Ciocio & $10 / 27 / 2004$ 11:13:00 PM \\
\hline $\begin{array}{l}\text { Page 3: [10] Deleted } \\
\quad ;\end{array}$ & A. Ciocio & $10 / 27 / 2004$ 11:13:00 PM \\
\hline $\begin{array}{l}\text { Page 3: [10] Deleted } \\
\text { the }\end{array}$ & A. Ciocio & $10 / 27 / 2004$ 11:13:00 PM \\
\hline $\begin{array}{l}\text { Page 3: [10] Deleted } \\
\quad \text { C }\end{array}$ & A. Ciocio & $10 / 27 / 2004$ 11:13:00 PM \\
\hline
\end{tabular}




\begin{tabular}{|c|c|c|}
\hline Page 3: [10] Deleted & A. Ciocio & 10/ 27/ 2004 11:14:00 PM \\
\hline \multicolumn{3}{|l|}{$\mathrm{S}$} \\
\hline Page 3: [10] Deleted & A. Ciocio & 10/ 27/ 2004 11:14:00 PM \\
\hline \multicolumn{3}{|l|}{ is } \\
\hline Page 3: [10] Deleted & A. Ciocio & $10 / 7 / 2004$ 10:49:00 AM \\
\hline \multicolumn{3}{|l|}{ ABCD3T } \\
\hline Page 3: [11] Deleted & A. Ciocio & 10/ 27/ 2004 11:10:00 PM \\
\hline \multicolumn{3}{|l|}{ the } \\
\hline Page 3: [11] Deleted & A. Ciocio & 10/ 27/ 2004 11:10:00 PM \\
\hline \multicolumn{3}{|l|}{ W } \\
\hline Page 3: [11] Deleted & A. Ciocio & 10/ 27/ 2004 11:10:00 PM \\
\hline \multicolumn{3}{|l|}{$\mathrm{W}$} \\
\hline Page 3: [12] Deleted & A. Ciocio & $10 / 7 / 2004$ 10:49:00 AM \\
\hline \multicolumn{3}{|l|}{ ABCD3T } \\
\hline Page 3: [12] Deleted & A. Ciocio & $10 / 27 / 2004$ 11:15:00 PM \\
\hline \multicolumn{3}{|l|}{, } \\
\hline Page 3: [12] Deleted & A. Ciocio & 10/ 7/ 2004 12:48:00 PM \\
\hline Page 3: [13] Deleted & A. Ciocio & $10 / 7 / 2004$ 10:48:00 AM \\
\hline \multicolumn{3}{|l|}{ ABCD3T } \\
\hline Page 3: [13] Deleted & A. Ciocio & 10/ 28/ 2004 4:38:00 PM \\
\hline \multicolumn{3}{|l|}{$\&$} \\
\hline Page 3: [13] Deleted & A. Ciocio & 10/ 6/ 2004 3:34:00 PM \\
\hline \multicolumn{3}{|l|}{$\mathrm{P}$} \\
\hline Page 3: [14] Deleted & A. Ciocio & 10/ 27/ 2004 11:11:00 PM \\
\hline \multicolumn{3}{|l|}{-} \\
\hline Page 3: [14] Deleted & A. Ciocio & 10/ 27/ 2004 11:11:00 PM \\
\hline \multicolumn{3}{|l|}{-} \\
\hline Page 3: [14] Deleted & A. Ciocio & 10/27/ 2004 11:12:00 PM \\
\hline \multicolumn{3}{|l|}{$\mathrm{P}$} \\
\hline Page 4: [15] Deleted & A. Ciocio & 10/ 6/ 2004 9:44:00 PM \\
\hline
\end{tabular}

A "Strobe Delay Scan" is performed as the very first of the analogue test sequence to determine the correct Strobe Delay setting, corresponding to the timing of the charge injection pulse, and it is used during the analogue tests.

Page 5: [16] Deleted A. Ciocio 9/ 23/ 2004 10:53:00 PM

The operating point for the Strobe Delay is chosen as the $25 \%$ of the distance between the $50 \%$ efficiency of the rising edge and the falling edge of the $4 \mathrm{fC}$ signal at $2 \mathrm{fC}$ threshold when varying delay values.
Page 5: [17] Deleted
A. Ciocio
10/ 28/ 2004 11:00:00 AM

A small but relevant percentage $(<5 \%)$ of IC's that met all the specifications at the level of wafer testing, developed a new set of defects after being mounted on hybrids during the hybrid/module production phase. Defects in the digital circuitry were considered as fatal and the chips were replaced while some of the defects in the analogue 
circuitry were studied to see if the ASICs would improve their performance by adjusting their front-end parameters.

$\begin{array}{ccc}\text { Page 5: [18] Formatted } & \text { A. Ciocio } & \text { 10/ 7/ 2004 9:58:00 PM } \\ \text { Font: Times New Roman, Not Bold } & & \end{array}$

$\begin{array}{lll}\text { Page 5: [19] Deleted } & \text { A. Ciocio } & \text { 9/ 18/ 2004 10:58:00 PM }\end{array}$

The capability

Page 5: [20] Formatted

Font: Times New Roman, 12 pt

\begin{tabular}{lll}
\hline Page 5: [21] Formatted & A. Ciocio & 10/ 7/ 2004 9:58:00 PM
\end{tabular}

Font: Times New Roman, 12 pt

Page 5: [22] Formatted

Font: Times New Roman, 12 pt

\begin{tabular}{ccc}
\hline Page 5: [23] Formatted & A. Ciocio & 10/ 7/ 2004 9:58:00 PM \\
Font: Times New Roman, 12 pt & & 10/ 7/ 2004 9:58:00 PM \\
\hline Page 5: [24] Formatted & A. Ciocio & \\
Font: Times New Roman, 12 pt & & 10/ 7/ 2004 2:37:00 PM \\
\hline Page 5: [25] Formatted & A. Ciocio & \\
Font: Times New Roman, 10 pt & & 10/ 28/2004 11:02:00 AM
\end{tabular}

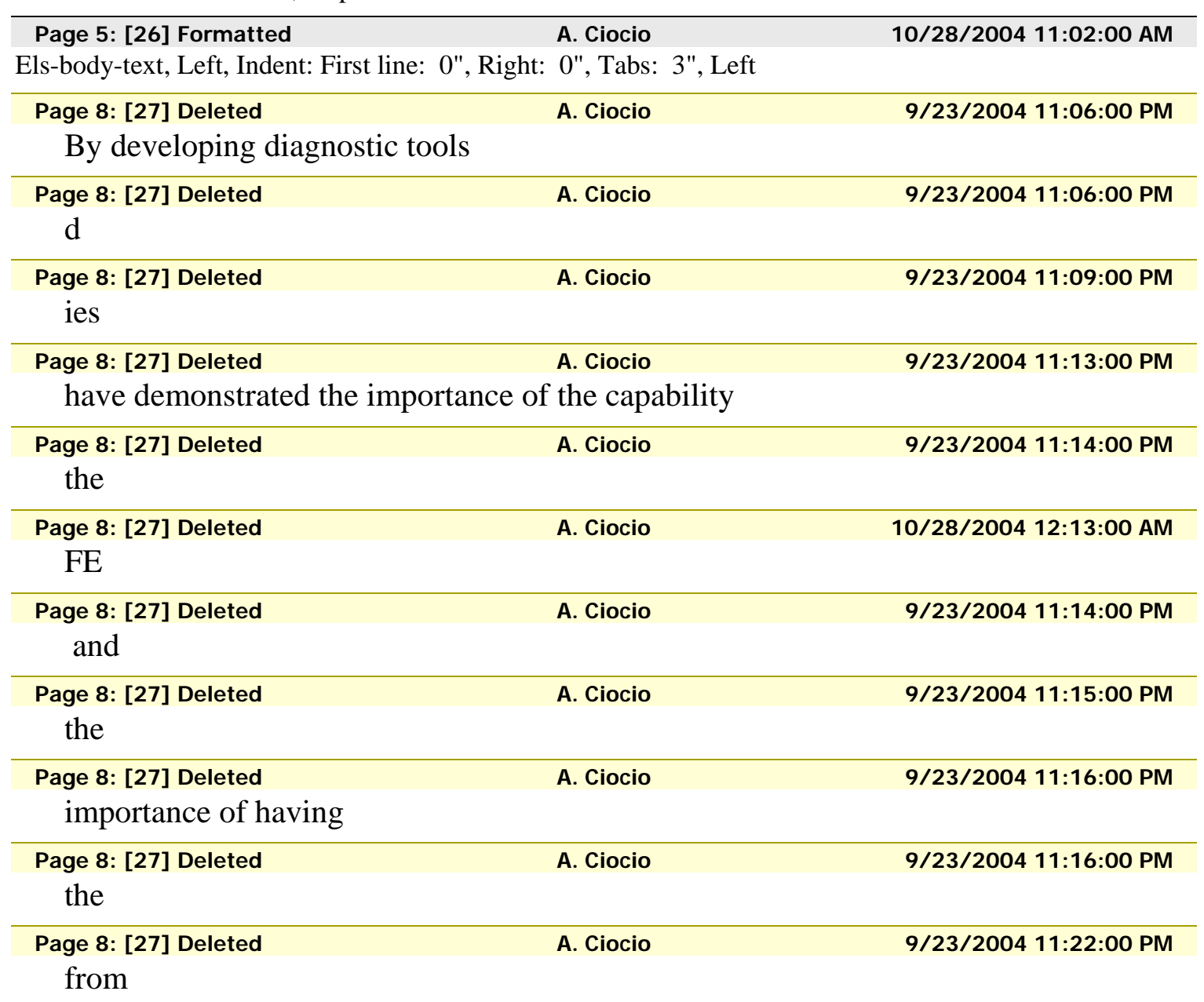




\begin{tabular}{|c|c|c|}
\hline Page 8: [27] Deleted & A. Ciocio & 9/23/2004 11:22:00 PM \\
\hline \multicolumn{3}{|l|}{ tests } \\
\hline Page 8: [28] Deleted & A. Ciocio & 10/ 6/ 2004 11:58:00 PM \\
\hline \multicolumn{3}{|l|}{2} \\
\hline Page 8: [28] Deleted & A. Ciocio & 10/ 9/ 2004 1:10:00 AM \\
\hline Page 8: [28] Deleted & A. Ciocio & $10 / 28 / 2004$ 12:12:00 AM \\
\hline \multicolumn{3}{|l|}{$\mathrm{S}$} \\
\hline Page 8: [28] Deleted & A. Ciocio & $10 / 28 / 2004$ 12:12:00 AM \\
\hline \multicolumn{3}{|l|}{$\mathrm{d}$} \\
\hline Page 8: [28] Deleted & A. Ciocio & 9/29/2004 9:35:00 AM \\
\hline \multicolumn{3}{|c|}{ The plateau in gain is not as flat as one might like in both case and } \\
\hline Page 8: [28] Deleted & A. Ciocio & 10/ 7/ 2004 11:52:00 AM \\
\hline \multicolumn{3}{|l|}{$\mathrm{t}$} \\
\hline Page 8: [28] Deleted & A. Ciocio & $10 / 7 / 2004$ 11:53:00 AM \\
\hline \multicolumn{3}{|l|}{6} \\
\hline Page 8: [28] Deleted & A. Ciocio & $10 / 7 / 2004$ 11:53:00 AM \\
\hline \multicolumn{3}{|l|}{, } \\
\hline Page 8: [28] Deleted & A. Ciocio & $10 / 7 / 2004$ 11:53:00 AM \\
\hline \multicolumn{3}{|l|}{ plateau } \\
\hline Page 8: [28] Deleted & A. Ciocio & 10/ 28/2004 12:13:00 AM \\
\hline \multicolumn{3}{|l|}{$\mathrm{S}$} \\
\hline Page 8: [28] Deleted & A. Ciocio & $10 / 28 / 2004$ 12:13:00 AM \\
\hline \multicolumn{3}{|l|}{$\mathrm{d}$} \\
\hline Page 8: [28] Deleted & A. Ciocio & $10 / 28 / 2004$ 12:13:00 AM \\
\hline \multicolumn{3}{|l|}{$\mathrm{S}$} \\
\hline Page 8: [28] Deleted & A. Ciocio & $10 / 28 / 2004$ 12:13:00 AM \\
\hline \multicolumn{3}{|l|}{$\mathrm{d}$} \\
\hline Page 8: [28] Deleted & A. Ciocio & $10 / 28 / 200412: 42: 00$ AM \\
\hline \multicolumn{3}{|l|}{$\mathrm{a}$} \\
\hline Page 8: [28] Deleted & A. Ciocio & $10 / 28 / 2004$ 12:18:00 AM \\
\hline \multicolumn{3}{|l|}{$\mathrm{S}$} \\
\hline Page 8: [28] Deleted & A. Ciocio & $10 / 28 / 2004$ 12:19:00 AM \\
\hline \multicolumn{3}{|l|}{$\mathrm{d}$} \\
\hline Page 8: [28] Deleted & A. Ciocio & 9/29/ 2004 9:36:00 AM \\
\hline \multicolumn{3}{|c|}{ becomes the same for all channels } \\
\hline Page 8: [28] Deleted & A. Ciocio & 10/ 28/2004 12:19:00 AM \\
\hline \multicolumn{3}{|l|}{ S } \\
\hline Page 8: [28] Deleted & A. Ciocio & 10/ 28/2004 12:19:00 AM \\
\hline \multicolumn{3}{|l|}{$\mathrm{d}$} \\
\hline Page 8: [29] Deleted & A. Ciocio & $10 / 28 / 2004$ 12:20:00 AM \\
\hline $\mathrm{S}$ & & \\
\hline
\end{tabular}




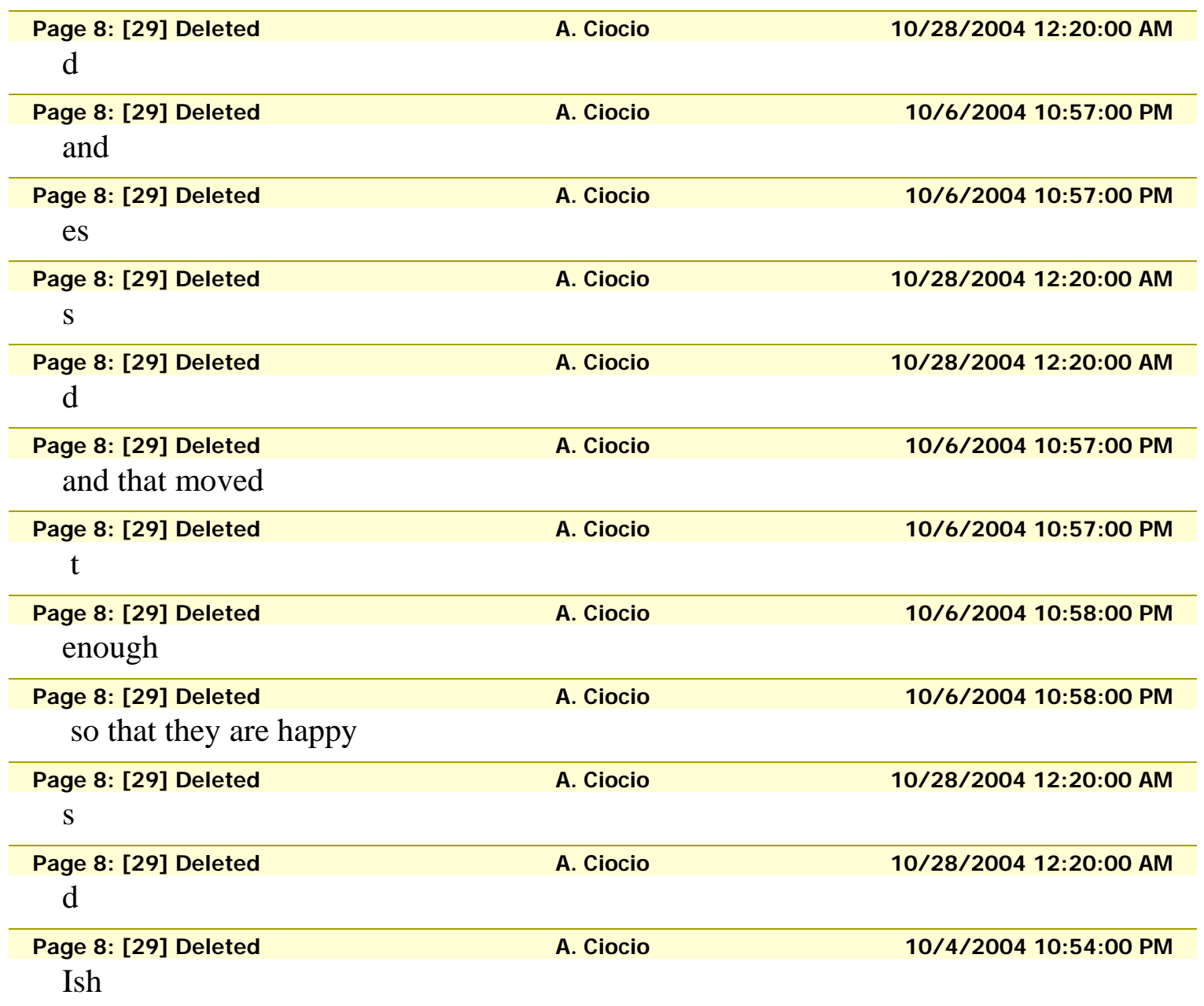

